\title{
Advanced NBI beam characterization capabilities at the recently improved test facility BATMAN Upgrade
}

\author{
U. Fantz*, F. Bonomo, M. Fröschle, B. Heinemann, A. Hurlbatt, W. Kraus, L. Schiesko, R. Nocentini, \\ R. Riedl, C. Wimmer
}

Max-Planck-Institut für Plasmaphysik, Boltzmannstr. 285748 Garching, Germany

\begin{abstract}
The test facility BATMAN was dedicated since its start in 1996 to the development of radio frequency driven negative hydrogen ion sources for ITER NBI with focus on formation and extraction of negative ions, technological developments and improved concepts. During 2017, the test facility was upgraded in order to replace the former extraction system with a new ITER-like extraction system comparable in size to one ITER beamlet group and having the option to flow a current through the grid creating a magnetic filter field as foreseen for the ITER sources. In addition, the beam diagnostics has been extended: beam emission spectroscopy is located at two positions from the grounded grid $(26 \mathrm{~cm}$ and $129 \mathrm{~cm})$ with spatial resolution in vertical direction. A newly developed tungsten wire calorimeter is placed just $19 \mathrm{~cm}$ downstream the grounded grid to provide quantitative measurements of individual beamlets, whereas the tungsten wire calorimeter at $180 \mathrm{~cm}$ distance is still in use for qualitative beam profile diagnostics. Together with a beam dump calorimeter with a crosswise arrangement of thermocouples, beam divergence and uniformity can be studied. This is accompanied by modeling the beamlet transport from the extraction system up to the calorimeter. Results from the first experimental campaign are reported, being very promising for detailed understanding of features measured on large beams.
\end{abstract}

Keywords: Neutral Beam Injection, ITER, negative ion source, radio frequency source.

\section{Introduction}

The development of a radio frequency $(R F)$ driven source for negative hydrogen ions for the neutral beam injection system of ITER has been successfully carried out at IPP since 1996 on the test facility BATMAN. The concept is based on the RF-driven positive ion source being reliably in operation at the NBI systems of ASDEX-Upgrade since more than 20 years and started recently at W7-X [1,2]. Due to the successful demonstration of the required current densities for $\mathrm{H}^{-}$ and for $\mathrm{D}^{-}$at the source pressure of $0.3 \mathrm{~Pa}$, the modular ion source concept became the prototype source for ITER NBI in 2007 [3,4].

Besides the task to achieve the required extracted current density of $329 \mathrm{~A} / \mathrm{m}^{2}$ for $\mathrm{H}^{-}$and $286 \mathrm{~A} / \mathrm{m}^{2}$ for $\mathrm{D}^{-}$ for short pulses ( $4.5 \mathrm{~s}$ beam within a $10 \mathrm{~s}$ plasma phase) reliably, the activities at BATMAN focused on the physics understanding of the formation and extraction of negative ions, and on testing new concepts for the improvement of the source performance. Therefore, the ion source is very well equipped with diagnostics and the compact size allowed for modifications in reasonable time and costs. The development was accompanied by the steady-state test facility MANITU targeting to achieve the parameters for the required long pulse operation (1000 s for $\mathrm{H}^{-}$and $3600 \mathrm{~s}$ for $\left.\mathrm{D}^{-}\right)$. Both ion sources have a source area of $1 / 8$ of the ITER source of $1 \times 2 \mathrm{~m}^{2}$. The latter has an extraction area of $0.2 \mathrm{~m}^{2}$ consisting of 1280 apertures. The extraction system, the so-called "Large Area Grid" (LAG) was derived from a positive ion accelerator from ASDEX Upgrade using its aperture size $(\varnothing 8 \mathrm{~mm})$ and pattern but replacing the first two electrodes and masking down the extraction area to
$70 \mathrm{~cm}^{2}$ (BATMAN) or $200 \mathrm{~cm}^{2}$ (MANITU). In 2011, operation of MANITU stopped because of the usage of components for the $1 / 2$ size ITER source test facility ELISE which started with the first experiments in 2013. Details of the development program and achievements can be found in $[3,5]$.

In order to produce ITER relevant beams and to focus the activities on the beam optics and the understanding of beam properties, the test facility BATMAN needed to be upgraded, for which operation stopped end of 2016. A central part of BATMAN Upgrade (BUG) is the new extraction system, which represents almost one of the beamlet groups of the ITER sources. For measuring the beam properties, a variety of diagnostic systems are implemented downstream of the extraction system. To study the influence of different magnetic filter field configurations on the beam properties, the option to have a current flowing through the plasma grid is provided, like the ITER sources. Further modifications concern the replacement of the self-excited RF generator by solid-state generators, the replacement of the electrical circuits and the cooling system resulting altogether in higher reliability of BATMAN Upgrade [6,7]. The test facility went into operation in June 2018 followed by a very promising operational campaign in hydrogen [7]. This paper focuses on the beam characterization capabilities and presents first results.

\section{BATMAN Upgrade}

\subsection{The test facility}

An overview of the test facility is shown in figure 1. The ion source itself has been reused from MANITU and 
is thus ready for steady-state operation. The plasma is generated in a cylindrical driver by inductive coupling of $\mathrm{RF}$ power using a solid-state based RF generator at 1 $\mathrm{MHz}$ frequency. At present, two generators are available, one with $75 \mathrm{~kW}$ and one with $150 \mathrm{~kW}$ maximum output power, the latter being currently connected. The plasma expands into the expansion chamber and illuminates the first grid of the extraction system. Ceasium is evaporated to produce sufficient negative hydrogen ions by the surface conversion process [8]. The Faraday screen in the driver and the expansion chamber are molybdenum coated. Additional diagnostic ports have been added to the expansion chamber allowing a video camera to be mounted to monitor the plasma structure during parameter variations, and performing optical emission spectroscopy to obtain the respective plasma parameters [9].

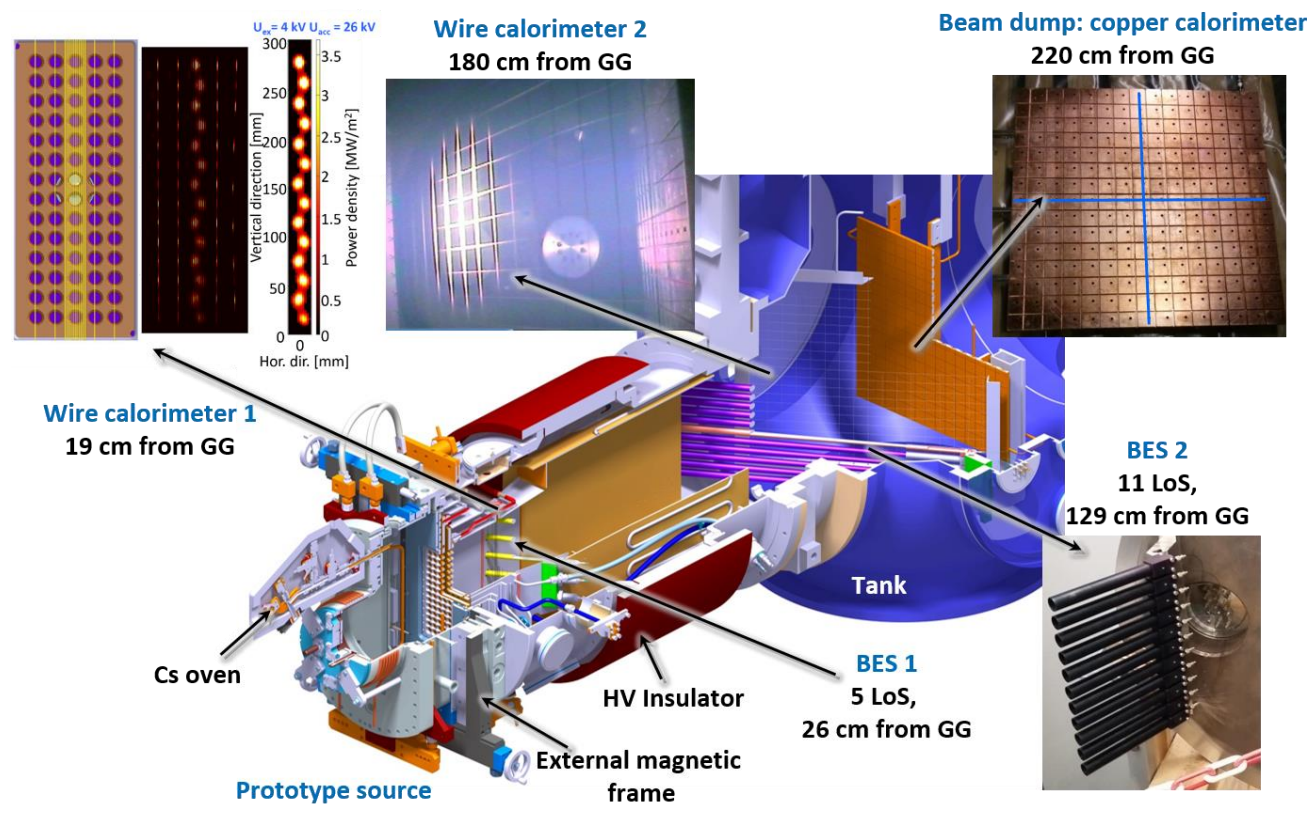

Fig. 1. The BATMAN Upgrade test facility with the beam diagnostic tools and their position relative to the last grid of the extraction system, the grounded grid (GG).

The entire extraction system of BATMAN is completely replaced, except the porcelain main insulator, the neutralizer and the ground support tube [6]. The main insulator separates the high voltage potential of the source from the ground potential of the test facility. The HV power supply is upgraded from the previous available $23 \mathrm{kV}$ in total to $50 \mathrm{kV}(15 \mathrm{~A})$ delivering 45 $\mathrm{kV}$ to the source as $5 \mathrm{kV}$ are used by the HV tubes. Up to $15 \mathrm{kV}$ are available for extraction. In order to withstand the higher acceleration voltage, gaps between structural components are increased and all feedthroughs have been redesigned.

The extraction system consists of a plasma grid, facing the plasma with a bias plate on top which is electrically connected to the source body, an extraction grid and the grounded grid (figure 2). Unlike the ITER extraction system, there is an additional fourth electrode located between the extraction and grounded grids. By applying a positive potential (up to $2 \mathrm{kV}$ ) with respect to the grounded grid, this repeller grid can be used for tests to reduce the space charge blow up of the beam and to suppress the current of back-streaming positive ions. At present, this grid is connected to ground potential.

The aperture pattern of the grids is identical to the ELISE pattern and very similar to one beamlet group of the ITER extractor. Only the number of apertures had to be reduced to $5 \times 14$ while it is $5 \times 16$ for one beamlet group of ELISE/ITER. This is caused by the geometrical limitations of the $\mathrm{HV}$ insulator and stacked grid holder boxes. The total extraction area is $108 \mathrm{~cm}^{2}$.

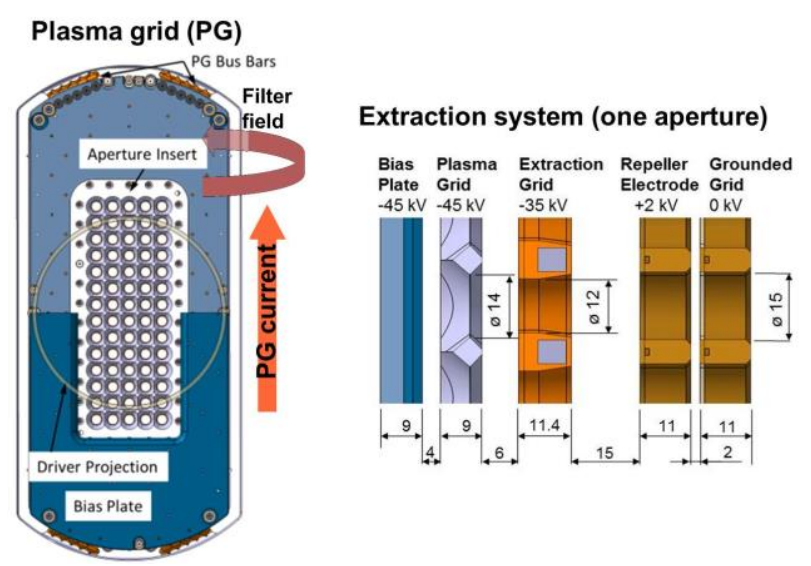

Fig. 2. View onto the plasma grid with the projection of the driver indicated. The extraction system is shown for one aperture; dimensions are in $\mathrm{mm}$.

All grids can be accessed for modifications from the source side and disassembled sequentially, without taking apart the entire grid structure as all water connections are screwed from the plasma side. A special feature is provided for the plasma grid: It is composed of two molybdenum plates screwed together with a heating wire clamped in between, and the aperture group is an 
independent insert, which allows easier modification of the aperture shape or grid material by only machining the central part and not the entire grid. The plasma grid can be heated up to temperatures of $\sim 800{ }^{\circ} \mathrm{C}$.

As negative ion sources require a magnetic filter field to cool down and to decrease the density of the electrons close to the plasma grid, the transverse field was generated by permanent magnets either embedded in the diagnostic flange or added to the lateral walls of BATMAN. In the upgrade, the filter field can now also be generated by a current flowing through the plasma grid ( $\mathrm{PG}$ current up to $3 \mathrm{kA}$, figure 2) which is the configuration of ELISE and ITER beam sources. This flexible approach allows studying the effects on the plasma drift and on the beam homogeneity.

At present, BATMAN Upgrade operates in pulsed mode, i.e. up to $10 \mathrm{~s}$ plasma pulses including up to $4.5 \mathrm{~s}$ beam pulses repeated every 3 minutes. Beam duration is limited by the pumping capacity of the titanium sublimation pumps and the inertially cooled copper calorimeter acting as beam dump. Cryo-pumps and a new actively cooled diagnostic calorimeter will be installed over the next months, to allow for steady-state operation.

\subsection{Beam diagnostic tools}

The beam diagnostics of BATMAN consisted of (see also figure 1): (i) the copper calorimeter at $220 \mathrm{~cm}$ distance from the grounded grid to measure the beam power by water calorimetry and the beam profile by thermo-couples embedded in the horizontal and vertical axis, (ii) the tungsten wire calorimeter at $180 \mathrm{~cm}$ to monitor the beam shot by shot, and, (iii) the beam emission spectroscopy (BES) at $129 \mathrm{~cm}$ with 5 lines-ofsight (LOS) to determine the beam intensity and the beam divergence vertically resolved [10].

Six additional lines of sight have been installed observing the beam at $129 \mathrm{~cm}$, for a total of 11 . With a vertical spacing of $3 \mathrm{~cm}$ they cover the central $30 \mathrm{~cm}$ of the beam, and observe from an angle of $57^{\circ}$ with respect to the beam propagation. These lines of sight are collectively known as BES 2, as a second array of 5 lines of sight (BES 1) has been installed observing the beam at $29 \mathrm{~cm}$ from the grounded grid. BES 1 is also arranged vertically, with a spacing of $6 \mathrm{~cm}$, and at the angle of $57^{\circ}$. Although the spatial resolution is lower than BES 2, the proximity to the extraction system means less crosstalk between aperture rows will have occurred, which should allow BES 1 to give a clearer picture of any beam inhomogeneity. Both arrays are connected to the same spectroscopic system that can accommodate 11 lines of sight simultaneously, with a spectral resolution of $19 \mathrm{pm}$.

A new tungsten wire calorimeter is placed much closer to the grid with one wire in the propagation of each beamlet column except the center column for which 11 wires are used [11]. Hence, the footprint of the beam observed by a camera offers the unique possibility to measure individual beamlets and to obtain the uniformity in the columns of the beamlet group. The option to heat one wire for calibration purposes even allows for the measurement of absolute power densities.

To help to establish links between diagnostic results and beamlet behavior, the particle tracking code BBCNI was developed to forward calculate simulated diagnostics from particle level data, including BES and calorimeter measurements [12]. Full traceability of these synthetic diagnostics allows features to be linked to specific particle populations and areas of the beam, providing a much-needed understanding of experimental results.

Although electric and magnetic fields cannot be calculated self-consistently, as much information is included as possible. Magnetic fields are included for the whole simulation domain, from plasma volume to calorimeter, and individual 3D electric field maps are specified for each aperture.

\section{Results of the first experimental campaign}

The first experimental campaign of BATMAN Upgrade started with a Cs-free source in hydrogen followed by the Cs conditioning phase. Although a full ion source performance was not achieved in the short campaign (about 6 weeks only), typical extracted current densities of around $200 \mathrm{~A} / \mathrm{m}^{2}$ at an ion-to-electron ratio below one using $70 \mathrm{~kW} \mathrm{RF}$ power and about $9 \mathrm{kV}$ extraction voltage allowed for some first insights by the comprehensive beam diagnostics [7].

Although the HV conditioning of the grids to the maximum voltage available could not be performed in the limited time, a minimum beam divergence of $1.5^{\circ}$ (half e-folding width of the Doppler peak in BES spectra) was demonstrated with BES 2 for values of extraction and acceleration voltage of $6 \mathrm{kV}$ and $32 \mathrm{kV}$, respectively. This is much lower than the achieved minimum of $3^{\circ}$ at BATMAN and is similar to the values achieved at ELISE. At best performance it is expected that the measured divergence decreases below the $1.5^{\circ}$ so far achieved. For the ITER sources the target value is $7 \mathrm{mrad}\left(0.4^{\circ}\right)$ achieved by a different acceleration system and higher acceleration voltages.

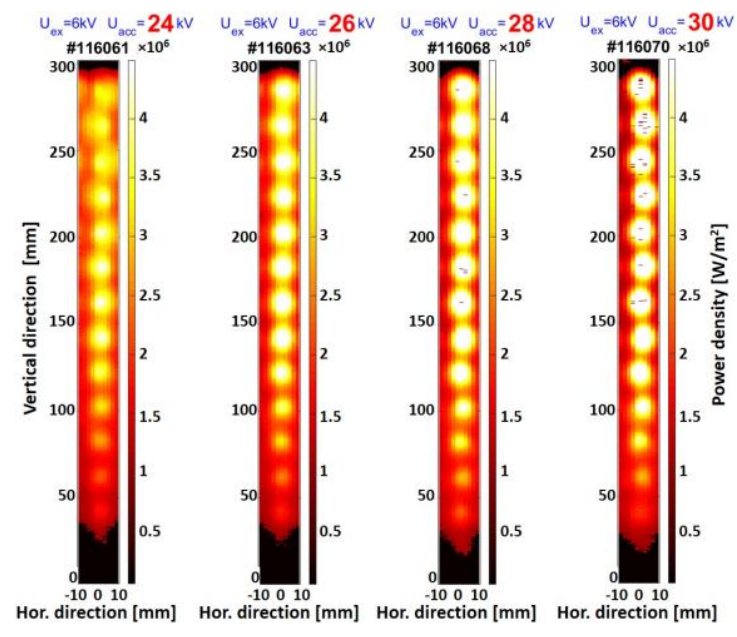

Fig. 3. Images from the wire calorimeter showing the central beamlet column for increasing acceleration voltages at a fixed extraction voltage of $6 \mathrm{kV}$. 
With the new tungsten wire calorimeter the individual beamlets are resolved and power densities are measured, as illustrated in figure 1 and discussed in [11]. The zig-zag pattern of the beamlets from row-to-row is caused by electron deflection magnets embedded in the extraction grid, arranged such that the polarity of the vertically arranged magnetic field alternates row-by-row. Hence, co-extracted electrons are dumped on the extraction grid whereas negative ions are slightly displaced horizontally.

Figure 3 gives an example of the change of the zigzag pattern by varying the acceleration voltage in steps of $2 \mathrm{kV}$ at fixed extraction voltage for consecutive pulses. The change of the zig-zag direction (pronounced between $26 \mathrm{kV}$ and $30 \mathrm{kV}$ ) is observed for the first time. A detailed analysis accompanied by BBCNI calculation is underway [12]. Figure 3 reveals also non-uniformities in the vertical power density. They depend on the characteristics of the source operation, such as caesium distribution and magnetic filter field intensity and topology.

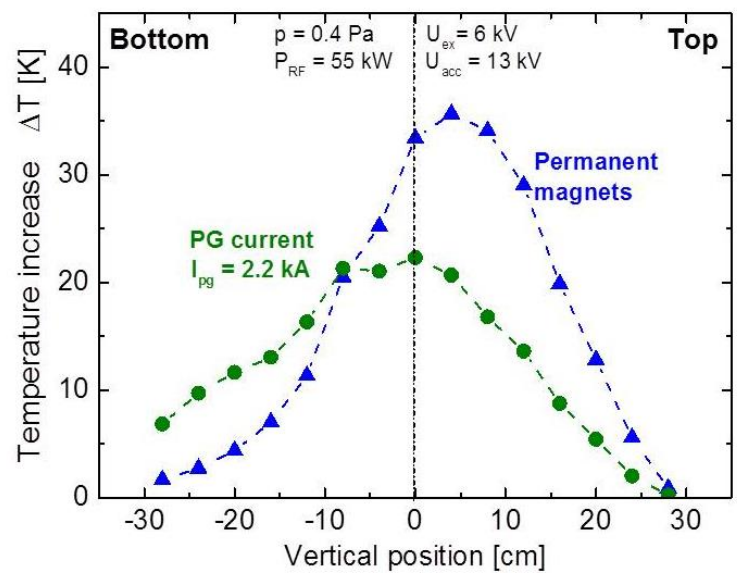

Fig. 4. Beam profile measured with the thermocouples in the calorimeter for the filter field created by the PG current and by permanent magnets.

The comparison of the two magnetic filter field cases (PG current or permanent magnets) shows an improved beam uniformity in terms of divergences for the $P G$ current as detected by BES 2 [7]: variations stay between $2.2^{\circ}$ and $2.8^{\circ}$ whereas the spread ranges from $1.5^{\circ}$ to $3.5^{\circ}$ in case of permanent magnets $\left(\mathrm{U}_{\mathrm{ex}}=6 \mathrm{kV}, \mathrm{U}_{\mathrm{acc}}=25\right.$ $\mathrm{kV}$ ). Figure 4 gives the vertical beam profile measured by the thermocouples in the calorimeter for the two cases of the filter field. The influence of the residual filter field in the accelerator is clearly detectable: the beam is deflected upward in case of permanent magnets whereas the deflection is downward for the PG current case. Deviations from a Gaussian profile are caused by the non-uniformity of the beam intensity as already indicated by the wire calorimeter.

Shown in figure 5 is an example of the diagnostic output of the BBNCI code, i.e. the vertical profile of normalized power density compared to the normalized profile measured by the calorimeter in the PG current configuration. Size and shape of the two curves are similar, but there is a difference in the apparent beam position. This is is most likely a result of BBCNI considering only uniform extracted current densities for all beamlets. In a next step, a vertical non-uniformity will be implemented in the calculation such that the profile can be obtained by the best match to experimental data.

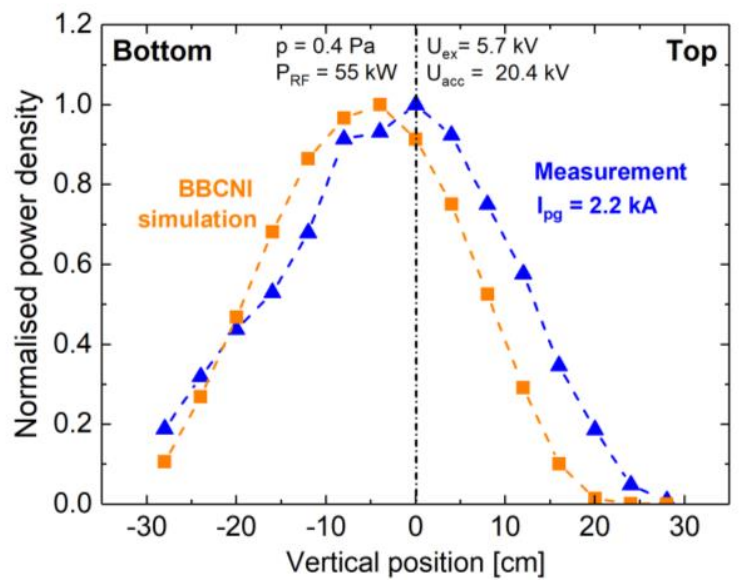

Fig. 5. Beam profile measured with the thermocouples in the calorimeter compared with BBCNI simulation for the magnetic field created by the PG current.

\section{Summary and Conclusions}

The BATMAN test facility has been successfully upgraded to study the characteristics of the beam by multifaceted beam diagnostics at ITER relevant extraction properties and a divergence comparable to the one achieved at ELISE. The latter is advantageous for improved data analysis at ELISE, as ELISE has reduced beam diagnostics and a much larger beam. Hence, BATMAN Upgrade is expected to give valuable input for the size-scaling experiment. The interpretation of the diagnostics is supported by the in-house developed particle tracking code BBCNI. The first experimental campaign was very promising giving already first insights in the uniformity of the total beam but also on the scale of individual beamlets. Furthermore the comparison of the two filter field configurations indicate improved uniformity for the PG current version.

\section{References}

[1] O. Vollmer et al., Commissioning and Performance of the New ASDEX Upgrade Neutral Beam Injector, Proc. 20th Symp. on Fusion Technology, (Marseille, 1998) 915.

[2] E. Speth et al., RF sources for Fusion Applications: Design, Development and Performance, Proc. 20th Symp. on Fusion Technology, (Marseille, 1998) 27.

[3] E. Speth et al., Overview of the RF source development programme at IPP Garching, Nucl. Fusion 46 (2006) 220.

[4] R. Hemsworth et al., Status of the ITER heating neutral beam system, Nucl. Dusion 49 (2009) 045006.

[5] B. Heinemann et al., Towards large and powerful radio frequency driven negative ion sources for fusion, New $\mathrm{J}$. Phys. 19 (2017) 015001.

[6] B. Heinemann et al., Upgrade of the BATMAN test facility for H- source development. AIP Conf. Proc. 1655 (2015) 060003.

[7] W. Kraus et al., First Beam Extraction Experiments at 
BATMAN Upgrade, AIP Conf. Proc. submitted.

[8] M. Bacal and M. Wada, Negative hydrogen ion production mechanisms, Applied Physics Reviews. 2 (2015) 021305.

[9] U. Fantz et al., Spectroscopic Investigations of the Ion Source at BATMAN Upgrade, AIP Conf. Proc. submitted.

[10] E. Aza et al., Beam Characterization at BATMAN for Variation of the Cs Evaporation Asymmetry and Comparing Two Driver Geometries, AIP Conf. Proc. 1869 (2017) 030037.

[11] R. Nocentini et al., A New Tungsten Wire Calorimeter for the Negative Ion Source Testbed BATMAN Upgrade, Fus. Eng. Des., submitted.

[12] A. Hurlbatt et al., The Particle Tracking Code BBCNI for Negative Ion Beams and its Application to BATMAN Upgrade, AIP Conf. Proc., submitted. 